\title{
L'impératif du bien-être: réflexion critique sur la notion de santé
}

\author{
La notion de santé tend à englober tous les aspects du bien-être et de l'épanouisse- \\ ment personnel. Peut-on dès lors concevoir que celui qui s'accommode de ses mala- \\ dies et des imperfections de sa condition humaine puisse encore s'affirmer heureux \\ et en bonne santé?
}

\section{Yves Rossier}

Directeur de l'Office fédéral des assurances sociales

Veuillez lire aussi à ce sujet I'article de Constantin Schuler (p. 1299) et le commentaire de Hans Kurt (p. 1303).
L'auteur remercie Madame Géraldine Luisier pour son précieux soutien lors de la rédaction de ce texte.

\section{Correspondance:} Yves Rossier Office fédéral des assurances sociales Effingerstrasse 20 CH-3003 Berne
«Click here for getting your health problems away at once!»: c'est le libellé d'un spam reçu à l'OFAS au moment de la préparation de cet exposé. Le message a le ton du défi: «If you are full of health, don't click!»

Et qu'auraient répondu en d'autres temps, d'autres lieux, nos prédécesseurs - ceux dont la vie s'épuisait dans la survie, ceux d'avant Louis Pasteur et l'irruption des microbes maléfiques, ceux qui ne savaient rien du subconscient ni des complexes, ceux d'avant la pollution et le spectre du poulet contaminé, ceux d'avant les «médicaliments» survitaminés et la santé à portée de clic de souris? de Baudelaire, pas de remède contre l'humanité de l'homme.

\section{Notion de santé}

Revenons tout d'abord à l'évolution de la notion de santé.

$\mathrm{Au}$ fur et à mesure que les besoins fondamentaux en nourriture et sécurité sont satisfaits, les aspirations portent sur d'autres dimensions qui viennent s'intégrer à l'idée de santé. Il ne s'agit plus de se contenter d'une bonne digestion et de se tenir droit sur ses jambes, sens premiers des expressions «bien aller» et

\section{Dernière étape en date de l'évolution de la notion de santé, après le bien-être complet de la définition de l'OMS: le mieux-être}

Pas de passéisme ou d'éloge de l'ignorance dans cette question - simplement un constat: le champ de la santé évolue, la place que celle-ci occupe dans la société et la vie des individus aussi. De la maladie punition divine au droit à la santé, il y a un progrès indéniable. Mais lorsque la santé devient un dû exigible à tout prix, avec pour corollaire le devoir d'être en forme et performant - «l'euphorie perpétuelle» de Pascal Bruckner -, il y a de quoi s'interroger. C'est ce que je me propose de faire avec vous, à travers une réflexion sur la notion de santé, puis sur le rapport entre santé et normalité dans la société actuelle. Cette réflexion sur la santé, si intimement liée à la conception de la vie et de l'homme, ne saurait toutefois s'épuiser dans le médical, ni dans le social. Aussi souhaiterais-je rappeler au cœur de notre propos d'aujourd'hui une dimension évacuée du discours actuel sur le rapport à la santé: le tragique inhérent à l'existence - il faudrait presque plaider pour un droit au tragique - la part de la condition humaine avec son imperfection et sa finitude. Soignons, apaisons les souffrances, améliorons les conditions de vie ici et ailleurs dans le monde. Mais pas, surtout pas, d'antalgique pour Antigone, pas de Prozac pour le spleen «bien se porter», mais d'accéder à un équilibre de vie, à la réalisation de soi. En passant d'une définition négative d'absence de maladie à une conception positive de bien-être ou de qualité de vie, la santé quitte simultanément le domaine réservé de la médecine pour relever d'une foule de disciplines et méthodes diffuses.

En effet, à l'époque moderne, la découverte des microbes et donc des mérites de l'hygiène, des vaccins et de la prévention permet d'entrevoir la possibilité d'une vie sans maladies. La foi dans le progrès scientifique, à partir du $19^{\text {e }}$ siècle, fait peu à peu sortir l'immortalité et la jeunesse éternelle des grands mythes où elle «donnait à penser» (P. Ricœur) à la condition de l'homme pour en faire des illusions suffisamment crédibles pour susciter des aspirations réelles.

Les progrès de la médecine, capable d'éradiquer presque complètement certaines maladies comme de découvrir l'influence de nouveaux facteurs sur le somatique, conduisent à adopter une vision plus large et positive de la santé dans l'après-guerre. C'est ce que fait l'OMS en 1946 en formulant la définition suivante: «La santé est un état complet de bien-être physique, mental et social qui ne consiste pas seulement en l'absence de maladie ou d'infirmité.» 
Arrêtons-nous un instant sur cette définition qui fait autorité. Avec elle, l'OMS poursuivait le louable objectif d'amener tous les peuples au niveau de santé le plus élevé possible. La définition présente un intérêt certain, en particulier dans le contexte des relations internationales:

- Elle insiste sur les différentes dimensions de la santé. En envisageant l'aspect physique, psychique et social, elle ne privilégie pas l'aspect purement somatique auquel se limite trop souvent l'horizon médical. Ce faisant, elle «démédicalise» la santé.

- C'est aussi une définition exigeante et dynamisante, car elle fixe un objectif très ambitieux. Elle reflète notre volonté, bien compréhensible, de repousser les frontières de la souffrance et de nous consacrer, selon les termes de la constitution américaine, à la poursuite du bonheur.

Cependant une telle acception de la santé comporte aussi des aspects critiquables:

- Elle pousse la démédicalisation très loin en insistant sur l'aspect purement subjectif de la santé. C'est le sujet et lui seul qui se dit ou non en bonne santé selon son ressenti, puisque c'est son bienêtre personnel qui définit la santé. Cette perception, bien sûr, est importante: on sait tous l'influence sur la maladie que peut avoir le vécu de la maladie. Mais elle peut aussi amener à un décalage entre mesure objective et perception subjective de la santé, voire à une impossibilité de communiquer, puisque, à la limite, chacun est sa propre référence. A titre d'exemple, on peut s'étonner que des personnes âgées handicapées, des malades chroniques lourds jugent souvent

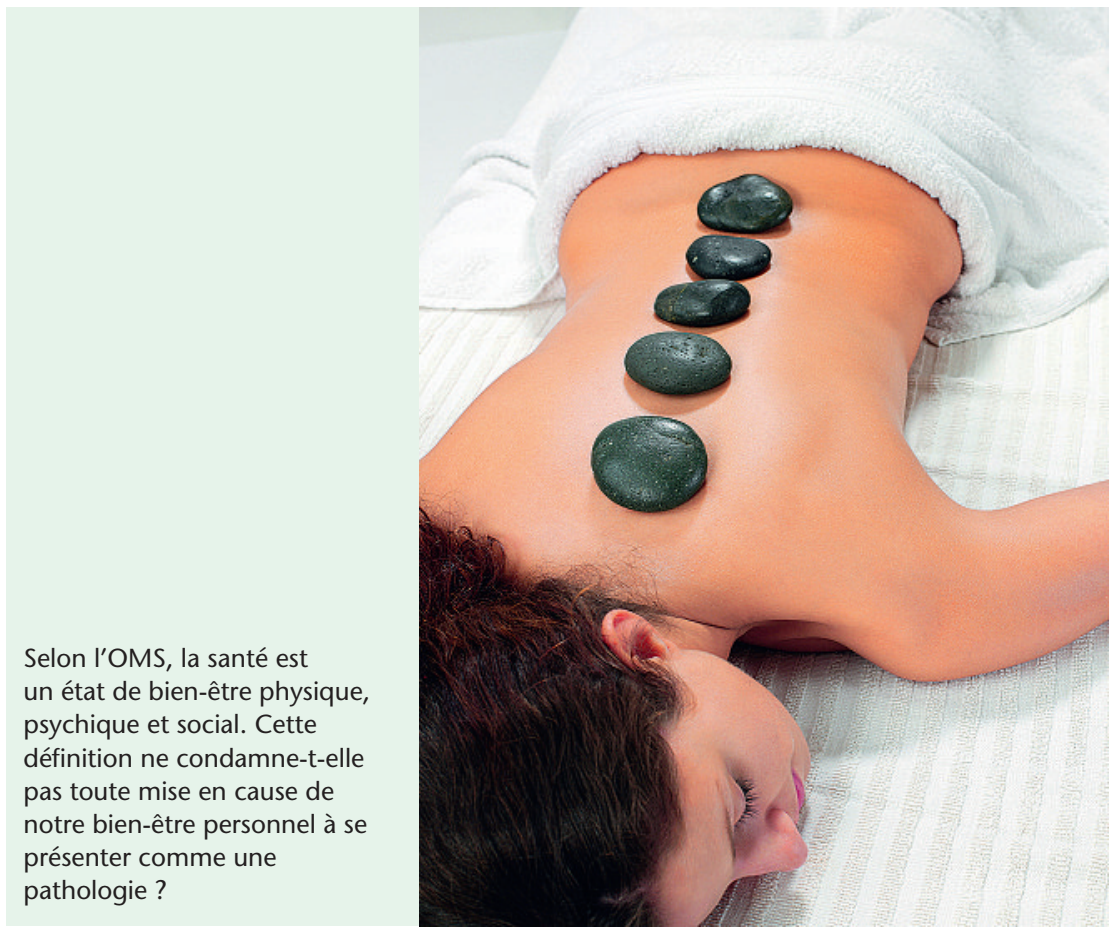

leur état de santé satisfaisant, l'inverse étant aussi communément observable.

- Cette définition pousse l'exigence d'être en parfaite santé jusqu'à l'utopie. Le bien-être «complet» existe-t-il parmi les vivants? Comment pourraiton le connaître, le mesurer? Prise à la lettre en effet, cette définition conduit à «fabriquer» plusieurs milliards de malades.

- Enfin, la définition de l'OMS est-elle à prendre comme une «vision» ou comme une prétention fondée en droit? S'il s'agit bel et bien d'un droit au bien-être, alors l'individu est en mesure de le faire valoir, non seulement à l'égard du corps médical, mais aussi à l'égard de son entourage, de son lieu de travail et de la société tout entière.

Le droit à la santé constitue un progrès incontestable. Il n'est cependant pas immunisé contre le risque d'être compris, dans nos sociétés de confort, comme une prétention opposable aux systèmes de santé et de sécurité sociale, comme un droit non seulement à être traité, mais à être guéri.

Deux dérives sont alors possibles dans la poursuite d'une même obsession de la santé parfaite:

- celle de «l'entreprise médicale», qui crée sans cesse de nouveaux besoins de soins, comme le critique Ivan Illich («Némésis médicale. L'expropriation de la santé», 1981); plus l'offre est pléthorique, plus le manque de santé est ressenti. Le marché de la santé accroît la demande de services pharmaceutiques et chirurgicaux et absorbe les ressources publiques au-delà du raisonnable.

- L'autre dérive, en partie en réaction à celle du tout scientifique et du tout médical, est dans la recherche tous azimuts de santé et même de guérison, jusque dans l'irrationnel. La perception d'un manque dans la «réalisation de soi» devient alors un état pathologique, qu'il convient de traiter et de guérir.

Dernière étape en date de l'évolution de la notion de santé, après l'absence de maladie et le bien-être complet de la définition de l'OMS: le mieux-être. Ainsi la demande de santé devient désormais une demande de corps performant, jeune et beau. On peut parler de «médecine du désir»: avec la chirurgie esthétique, les applications de la génétique, la médicamentation des problèmes sociaux, voici l'apparition des bien portants médicalement traités.

Le droit à être guéri, les possibilités offertes par la médecine du désir impliquent que la santé - identifiée avec la réalisation des désirs - est la norme. La maladie, voire toute imperfection, devient inacceptable, sans parler de la mort. Nous aboutissons ainsi à la négation même de la condition humaine, imparfaite et finie. Mais avant de développer ce thème, j'aimerais rester un instant sur la notion de norme ou de normal. 


\section{Le normal, le pathologique, la différence}

Comme le concept lui-même, la relation de la société avec la notion de santé et de maladie est une construction qui varie dans l'espace et le temps. Ainsi que le montrent anthropologues et sociologues, les interprétations et les pratiques sociales autour du normal et du pathologique, du sain et du malsain portent la marque des croyances et des idéologies.

Pour la médecine, à la suite d'Auguste Comte et Claude Bernard, le normal et le pathologique ne sont pas fondamentalement différents, ils ne présentent qu'une variation quantitative des phénomènes de l'organisme. Mais le pathologique n'en est pour lui pas moins normal en ce qu'il obéit à une normativité propre. «Être malade, c'est encore vivre, et vivre, c'est toujours fonctionner selon des normes, même restreintes; en outre, c'est vivre, parfois, selon une normativité toute nouvelle» (A. Kremer Marietti). Pour Canguilhem, le fond du problème réside dans la connaissance de la vie, qui ne saurait se réduire à un simple équilibre; la vie n'est pas «normale», même le pathologique est appelé à y participer.

Dans notre société, l'individu est le sujet à partir duquel tout se mesure. Ainsi la santé, dans la ligne de l'OMS, devient une question subjective de bien-être, de qualité de vie. N'est en bonne santé que celui ou celle qui se réalise soi-même, et pleinement. Telle est la norme. Mais se réaliser soi-même en fonction de quelle identité, en référence à quel idéal? La définition de la normalité reste l'affaire de la société et la référence dominante est aujourd'hui celle de la réalisation des désirs, de la consommation en quelque sorte. Le manque à combler pour parfaire son développement personnel est source d'attentes infinies, qu'attise l'offre quasi illimitée de produits désirables. Je suis en bonne santé si je suis en mesure, physiquement et financièrement, de réaliser l'ensemble des désirs qui me sont proposés.

\section{Le handicap jette une lumière crue et dérangeante sur le caractère illusoire de ce projet}

La référence au normal imprègne tout particulièrement la relation au handicap. Parce que le handicap est toujours une souffrance, et, qui plus est, une souffrance insurmontable, il est nécessairement une entrave à cette forme de réalisation personnelle. Le handicapé - ou le vieillard - de référence est celui qui participe pleinement à la société de consommation, qui fait du saut à l'élastique ou gravit le Kilimandjaro en solitaire. En revanche, qui se soucie de l'héroïsme de celui pour lequel le seul fait de se tenir debout est un exploit? Pire, par la visibilité de ses limites, le handicap jette une lumière crue et dérangeante sur le caractère illusoire de ce projet. Le handicap et toute forme de dégénérescence physique ou mentale sont donc à oblitérer. On ne peut s'empêcher donc de discerner une certaine contradiction dans une société qui bannit, à juste titre, toute forme de discrimination tout en mettant en place, par le diagnostic prénatal par exemple, les moyens de faire l'économie du sujet même de ces discriminations.

\section{La part de la condition humaine}

On ne peut s'en tenir à la dimension sociale de la santé, du rapport à la norme. La maladie, mais aussi la souffrance devant l'impossibilité du bien-être total renvoient immanquablement l'individu à un pourquoi. Elles le ramènent au tragique de la condition humaine, dans le sens d'un conflit entre les aspirations infinies de l'homme et ce qui ne dépend pas de lui, les limites de l'existence. Toutes les thérapies et autres bonheurs consommables guériront des pathologies, atténueront des douleurs, en évacueront la vision inesthétique mais n'élimineront ni la solitude, ni la peur, ni la mort.

L'individualisation des modes de vie et le poids accordé à la subjectivité font donc de chacun le responsable de sa réalisation personnelle, la norme étant la plénitude. À chacun de construire sa réponse à ce «pourquoi» existentiel sur le marché des idées.

Le bonheur semble s'ériger en idéologie. Il faut être heureux à tout prix, donc se soumettre à la tyrannie du bonheur, selon la belle formule de P. Bruckner. Au nom du plaisir immédiatement consommable, on tente de construire un monde aseptisé et euphorique, chimiquement pur de l'effort et des aléas qui jalonnent traditionnellement la route de la félicité et lui donnent son prix.

Comme le bonheur auquel elle est largement assimilée, la santé s'inscrit en valeur suprême. On peut alors dire avec le psychiatre et théologien Manfred Lütz que «l'homme sain devient l'homme vrai». Le culte de la santé et du corps parfait n'a-t-il pas une dimension quasi religieuse? Il a ses lieux de culte (hôpitaux, lieux de cures), ses rites (cures, fitness...), ses pélerinages (marathons..), ses sacrifices (régimes...), ses devoirs (alimentation saine, mouvement...), ses péchés (obésité, tabagisme...), et même sa mystique qui relève largement de l'irrationnel et de la crédulité. La poursuite de la santé, lorsqu'elle dérive vers l'obsession, s'inscrit tantôt dans une forme d'hédonisme (recherche du plaisir), tantôt dans une discipline stoïcienne.

L'assimilation du culte de la santé à une religion s'arrête ici. En effet, non seulement ce culte est sans transcendance, mais il ne propose aucune réponse aux questions fondamentales de l'homme sur lui-même, ou plutôt, sa réponse est dans le déni. Les signes du vieillissement, les troubles divers, la tristesse peuvent être maîtrisés, et même la mort peut s'aménager. Où est donc le problème? Le problème est que, selon les mots d'Ivan Illich, «la recherche de la santé est devenue le facteur pathogène prédominant». Autrement 
dit, loin de garantir le complet bien-être en occultant ce qui le trouble et le rend inatteignable, la société euphorique ouvre insidieusement la boîte de pandore de peurs et des angoisses existentielles.

En effet, derrière la recherche effrénée de la pleine réalisation de soi n’y a-t-il pas les ingrédients du tragique?

- La peur de la souffrance, de la dégénérescene et de la mort inéluctables, la peur du manque - une révolte muette contre la condition humaine, qu'elle soit imposée par les dieux, le destin ou les gènes.

- Le désarroi devant sa propre responsabilité; dans la société de précaution, il faut se prémunir des contaminations, s'immuniser pour limiter les risques. En matière de santé aussi, le patient doit «jouer son sort au poker», comme le dit encore Illich: on lui présente un diagnostic sous forme de courbes de probabilités et il lui appartient de «se risquer comme modèle actuariel pour décider de son traitement». On se souvient de l'affaire Perruche où la naissance d'un enfant handicapé donne lieu à une action en responsabilité et où l'on consacre l'expression de «vie préjudiciable».

- La culpabilité face au devoir d'être en forme; si la santé n'est plus un don de Dieu, il faut la mériter, l'acquérir, épuiser en quelque sorte les voies du droit à la santé. la valeur. La question du «pourquoi» et celle du «pour quoi» restent ici entières. Elles relèvent de la métaphysique ou de la théologie, et ce n'est pas mon propos de m'y exercer en cette occasion.

\section{Conclusion}

Il faut pourtant à ce point tenter une approche positive.

Nous avons critiqué la survalorisation de la santé «bien-être total». La définition de Nietzsche nous plaît: «Gesundheit ist dasjenige Mass an Krankheit, das es mir noch erlaubt, meinen wesentlichen Beschäftigungen nachzugehen.» Un homme est en bonne santé lorsqu'il est capable de vivre plus ou moins heureusement avec ses maladies. Ou pour le dire avec Freud: «Gesundheit ist die Fähigkeit, lieben und arbeiten zu können.»

Qui adopte cette approche pragmatique de la santé, libre d'attentes infinies, peut davantage accueillir la vie, tout simplement. Montaigne déjà déplorait que ses contemporains se troublent la vie par le souci de la mort, et la mort par le regret de la vie.

La condition humaine n'est pas qu'imperfection et finitude. Comme le mélange du grotesque et du sublime est le ressort de la dramaturgie romantique, l'existence est faite d'ombres et de lumières et vouloir trier entre elles en casse la dynamique. Gardons-nous

\section{Selon Nietzsche: Un homme est en bonne santé lorsqu'il est capable de vivre plus ou moins heureusement avec ses maladies}

La souffrance qui naît de l'inassouvi des aspirations, comme expérience du tragique de l'existence, n'est pas une pathologie; elle est bien plutôt un signe de santé, comprise cette fois non comme une réalisation complète de soi mais comme une saine relation de l'homme à sa condition: «Faire bien l'homme et dûment» (Montaigne).

Se souvenir que la maladie, la souffrance et la mort sont des éléments constitutifs de l'expérience humaine ne signifie nullement en faire l'apologie, en affirmer donc d'inviter Antigone à faire soigner ses obsessions et laissons les poètes à leur douleur d'habiter «avec un cœur plein un monde vide» (Chateaubriand).

Le tragique, dans les mythes comme dans la vie réelle, n'est possible que parce que l'homme est grand, capable d'amour, d'action, de création. Et si l'on en croit les caractères du théâtre de Giraudoux, même les dieux de l'Olympe envient pour cela les mortels, et ceux-ci, trop heureux de leurs petits bonheurs, n'échangeraient pour rien leur condition. 